\title{
Galactic Anisotropy of Cosmic Ray Intensity Observed by an Air Shower Experiment
}

\author{
Mahmud Bahmanabadi $^{\mathrm{A}, \mathrm{B}}$, Mehdi Khakian Ghomi ${ }^{\mathrm{A}}$, Farzaneh Sheidaei ${ }^{\mathrm{A}}$ and \\ Jalal Samimi $^{\mathrm{A}}$ \\ A Physics Department, Sharif University of Technology, P.O. Box 11365-9161, Tehran, Iran. \\ B Corresponding author. E-mail: bahmanabadi@sina.sharif.ac.ir
}

\begin{abstract}
We have monitored multi-TeV cosmic rays by a small air shower array in Tehran $\left(35^{\circ} 43^{\prime} \mathrm{N}\right.$, $51^{\circ} 20^{\prime} \mathrm{E}, 1200 \mathrm{~m}=890 \mathrm{~g} \mathrm{~cm}^{-2}$ ). More than $1.1 \times 10^{6}$ extensive air shower events were recorded. These observations enabled us to analyse sidereal variation of the galactic cosmic ray intensity. The observed sidereal daily variation is compared to the expected variation which includes the Compton-Getting effect due to the motion of the earth in the Galaxy. In addition to the Compton-Getting effect, an anisotropy has been observed which is due to a unidirectional anisotropy of cosmic ray flow along the Galactic arms.
\end{abstract}

Keywords: cosmic rays

\section{Introduction}

Cosmic rays have a remarkably uniform distribution over the sky, yet small anisotropies do exist. The deviation from isotropy is typically below $1 \%$ and can be as low as $0.03 \%$ (Smith \& Clay 1997). The galactic anisotropy of the cosmic-ray intensity is expected to carry information about the origin and the propagation mechanism of galactic cosmic rays, as it reflects the galactic magnetic field through which they have passed and their energy spectrum at source. It is presumed that the anisotropy at multi-TeV energies is almost free from heliosphere disturbance, while in the lower energy events the additional diurnal anisotropy is probably due to solar modulation. Cosmic rays in the lower energy range have gyro radii of about $1 \mathrm{pc}$ or less in typical galactic magnetic fields (a proton with an energy of $10^{15} \mathrm{eV}$ would have a gyro radius of $1 \mathrm{pc}$ in a $1 \mu \mathrm{G}$ field). Because of low anisotropy, it is assumed that cosmic ray propagation is diffusive in some way (Allan 1972). This diffusion may be broadly along the magnetic field lines which are in tubes of dimensions greater than the gyro radii. If so, then the direction of the peak of the anisotropy would indicate the direction back towards the cosmic ray source, and the amplitude of the anisotropy would give information on the scattering process involved in the diffusion. Specifically, an estimate of the mean free path might be obtained. A well known asymmetry effect is the Compton-Getting Effect (CGE). The earth's rapid motion in space due to the rotation of our galaxy, results in variations in cosmic ray intensity fore and aft of the earth's motion. Following Compton \& Getting (1935), the magnitude of the anisotropy is expressed as

$$
\eta=(\gamma+2) \frac{u}{c} \cos \theta
$$

where $\gamma$ denotes the power law index of the energy spectrum of cosmic rays, $u$ the velocity of the detector relative to the production frame of the cosmic rays (where they are presumed to be isotropic), $c$ the speed of light, and $\theta$ the cosmic ray direction relative to $u$, i.e. $\cos \theta$ is the projection of the cosmic ray along the forward direction of $u$. In fact the value of $(\gamma+2) \frac{u}{c}$ is $\left(f_{\max }-f_{\min }\right) /$ $\left(f_{\max }+f_{\min }\right)$ with $f_{\max }$ being the counting rate along the direction of the velocity and $f_{\min }$ the rate along the contrary direction. The magnitude of the anisotropy is extremely small and independent of the cosmic ray energy. Our data will be analyzed in a sun-centered frame, and so if data accumulation is done over an integer number of solar years, it is only necessary that the orbital speed of the Earth around the sun $\left(\sim 30 \mathrm{~km} \mathrm{~s}^{-1}\right)$ be considered; the large effect due to the Galactic rotational speed $\left(220 \mathrm{~km} \mathrm{~s}^{-1}\right)$ will cancel out as the data are averaged over this time (Poirier, D'Andrea \& Dunford 2001). Many experiments have been carried out for detection of the former effect (Poirier et al. 2001; Lin 1999).

Doppler effect studies of globular clusters and extragalactic nebulae have revealed a motion of the earth of about $220 \mathrm{~km} \mathrm{~s}^{-1}$ towards right ascension $\alpha \simeq 21 \mathrm{~h}$ and declination $\delta \simeq 47^{\circ} \mathrm{N}$, due chiefly to the rotation of the Galaxy. This motion, with a speed of about $0.1 \% c$, will affect the intensity of the incoming cosmic rays by changing both the energy of the cosmic ray particles and the number received per second. Using a value of 220 $\mathrm{km} \mathrm{s}^{-1}$ for $u$, and 2.7 for the spectral index, Eqn (1) gives a CGE amplitude of $0.345 \times 10^{-2}$ for the fractional forward-backward asymmetry caused by the motion of the earth in the Galaxy. Because of the small anisotropy, large data sets are required to make useful measurements which overcome the statistical uncertainties of counting experiments. A simple way of recording many cosmic rays is to record coincidences between a number of scintillation detectors. Few statistically significant anisotropics have been reported from extensive air shower experiments in the two last decades. Aglietta 
et al. (1996, EAS-TOP) published an amplitude of (3.7 \pm $0.6) \times 10^{-4}$ at $E_{\circ} \approx 200 \mathrm{TeV}$. Analyzing the Akeno experiment, Kifune et al. (1986) reported a result of about $2 \times 10^{-3}$ at about 5-10 PeV. An overview of experimental results can be found in Clay et al. (1997). We have operated a small air shower array on the roof of the Physics Department at Sharif University of Technology in Tehran $\left(35^{\circ} 43^{\prime} \mathrm{N}, 51^{\circ} 20^{\prime} \mathrm{E}\right.$, at $\left.1200 \mathrm{~m}=890 \mathrm{~g} \mathrm{~cm}^{-2}\right)$ as a prototype for constructing an Extensive Air Shower (EAS) array on Alborz mountain range ataltitude of over $2500 \mathrm{~m}$ near Tehran. At the time of writing, more than $1.1 \times 10^{6}$ showers have been recorded. In the present paper we discuss the design of this scintillator array and describe its operation. The main purpose of the experiment is to study the anisotropy due to the Compton-Getting effect and a unidirectional anisotropy of cosmic ray flow along the Galactic arms which was observed in the sidereal time at energies in the decade below $1 \mathrm{PeV}$.

\section{Experimental Setup}

Figure 1 shows an array including four slab plastic scintillators $\left(100 \times 100 \times 2 \mathrm{~cm}^{3}\right)$ arranged in a square with an edge size of about $10 \mathrm{~m}$ (Bahmanabadi et al. 2003). All scintillators are on a flat level surface. Each scintillator is housed in a pyramidal steel box with a height of $15 \mathrm{~cm}$. The interior surface of each box is coated with white paint

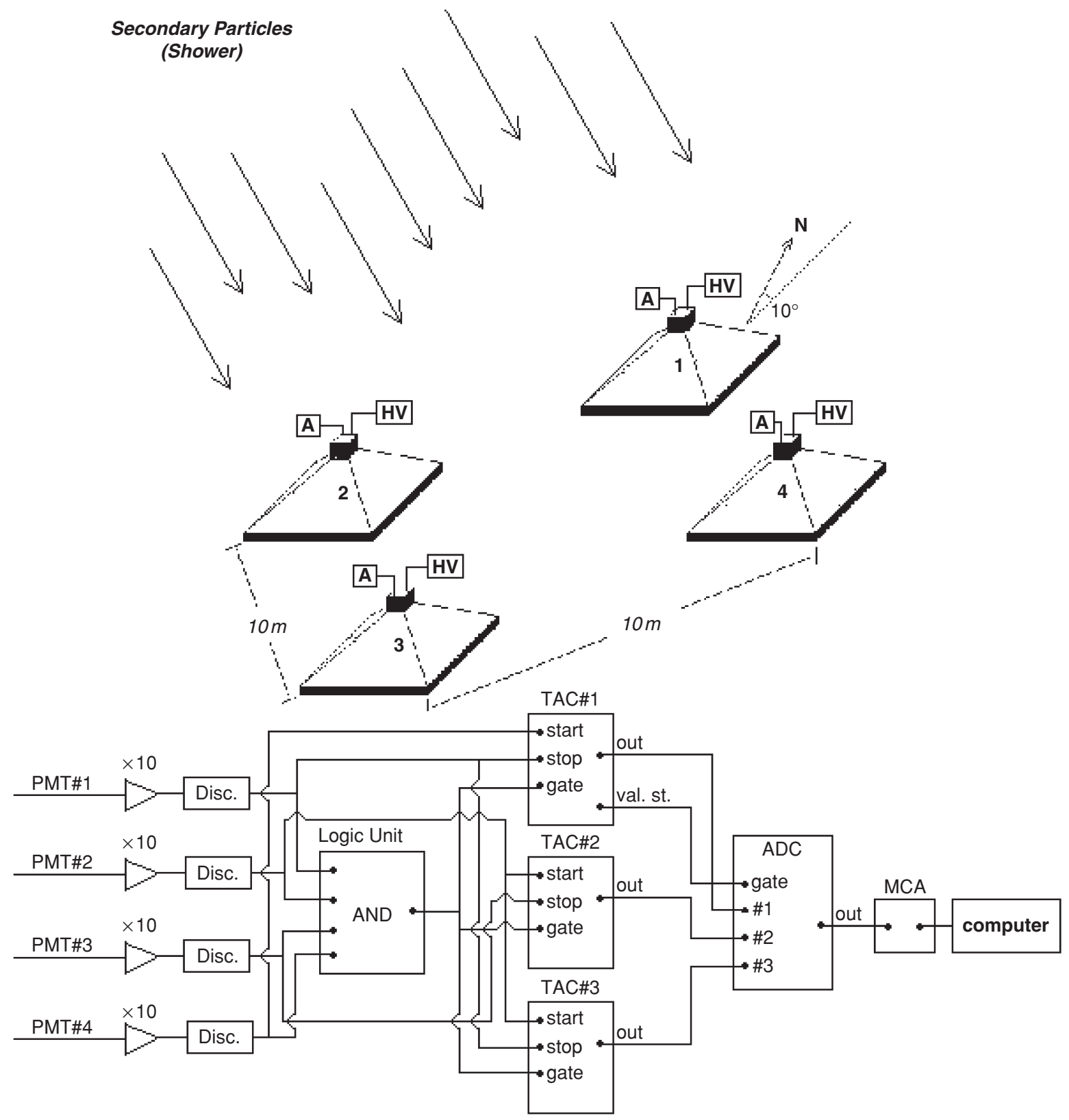

Figure 1 Arrangement of four scintillation detectors as a square array, and the electronic circuit. 
(Bahmanabadi et al. 1998) and a $5 \mathrm{~cm}$ diameter photo multiplier tube (PMT, EMI $9813 \mathrm{~KB}$ ) is placed at the vertex of the pyramidal box. Figure 1 shows a schematic diagram of the array and its electronic circuit which $\operatorname{logs}$ each EAS event. If at least one particle hits a detector, the PMT creates a signal with a pulse height which is related to the direction, the number of particles, and the location of the particle track in the scintillator. The output signals from the PMTs are amplified in a one-stage amplification $(\times 10)$ with an 8-fold fast amplifier (CAEN N412), and then transferred one by one to an 8-fold fast discriminator (CAEN N413A) used in leading edge mode which is operated at a fixed level of $20 \mathrm{mV}$. The threshold of each discriminator is set at the separation point between the signal and background noise levels. Each discriminator has two outputs, one of them connected to a coincidence logic unit (CAEN N455) which acts as a trigger condition. The trigger condition is satisfied when at least one charged particle passes through each of the four detectors within a time window of $150 \mathrm{~ns}$. The other discriminator output is connected to one of three Time to Amplitude Converters (TAC, EG\&G ORTEC 566) which are set to a full scale of $200 \mathrm{~ns}$ (maximum acceptable time difference between two scintillators). The output of scintillator \#4 is connected to the start input of TAC1, whereas the output of scintillator \#2 is connected to the start inputs of TAC2 and TAC3. The output of scintillator \#3 is connected to the stop input of TAC2, and that of \#1 to the stop inputs of TAC1 and TAC3. Finally the outputs of these three TACs are fed into a multi-parameter Multi Channel Analyzer (MCA, KIAN AFROUZ Inc.,) via an Analogue to Digital Converter (ADC, KIAN AFROUZ Inc.,) unit. When all of the scintillators have coincidence pulses, the TACs are trigged by the logic unit and the three time lags between the output signals of three pairs of the PMTs $(4,1),(2,3)$, and $(2,1)$, where the numbers in parentheses are the start and stop of the two TACs respectively, are read out by a computer and an EAS event is logged. All events with zenith angles $\leq 60^{\circ}$ are used in the present analysis. About $1.1 \times 10^{6}$ events remained after these selections.

\section{Data Analysis and Discussion}

Over a period of about 132 days (2002 Aug 01-Dec 10) we logged more than $1.1 \times 10^{6}$ events. The mean event rate of the experiment was 0.107 events per second. The distribution of the time between successive events is in good agreement with an exponential function, indicating that the event sampling was completely random (Bahmanabadi et al. 2003). We refined the data by selecting out acceptable events, i.e. those with good coincidence between the four scintillator pulses. To ensure reconstruction quality, only showers well inside the detector field with zenith angles smaller than $60^{\circ}$ have been used. After the selection, about $1.1 \times 10^{6}$ events remained for analysis. Further, only sidereal days with continuous data taking were used.

Since we cannot determine the energy of the showers on an event-by-event basis, we estimate the lower energy threshold by comparing our event rate to the well-known Hillas spectrum (Gaisser 1990), which can be represented as

$$
F(>E) \sim 2 \times 10^{-10} \frac{\text { particle }}{\mathrm{cm}^{2} \mathrm{~s} \mathrm{sr}} \times\left(\frac{E}{1000 \mathrm{TeV}}\right)^{-\gamma},
$$

with $\gamma=1.7$ for $E<1000 \mathrm{TeV}$.

We used CORSIKA (Heck et al. 1998), using the hadronic interaction models QGSJET and GHEISHA, to determine the surface such that, if a primary particle passes through it, our array could detect its EAS event. The energy range for primary particles was selected to be from $1 \mathrm{TeV}$ to $5 \mathrm{PeV}$, with differential flux given by $\mathrm{dN} / \mathrm{dE} \propto E^{-2.7}$. These simulations are in different directions, with zenith angles in bins $0^{\circ}-10^{\circ}, 10^{\circ}-20^{\circ}, \ldots$, and $50^{\circ}-60^{\circ}$, with all azimuth angles in each $10^{\circ}$ zenith angle interval. Since our scintillation detectors are $1 \mathrm{~m} \times 1 \mathrm{~m}$ in size, and if at least one particle passes through a detector it is sufficient to record a pulse, we used the condition of particle density greater than $1 \mathrm{~m}^{-2}\left(\rho \geq 1 \mathrm{~m}^{-2}\right)$ is used. Based on the NKG formula (Gaisser 1990), the mean effective surface of EAS events at Tehran level is about $2000 \mathrm{~m}^{2}$. With this result we estimate the mean effective surface area of our array in the upper level of the atmosphere (the surface such that if a primary particle passes through it, the array could detect its EAS event) to be about $1500 \mathrm{~m}^{2}$. The field of view of our array to zenith angle $60^{\circ}$ is $\pi$ steradian. Therefore, based on Eqn (2), the energy threshold is about $250 \mathrm{TeV}$. The mean energy of cosmic rays, calculated by the differential spectrum obtained by Eqn (2), is about $420 \mathrm{TeV}$.

If either temperature or pressure variations have Fourier components in sidereal time, spurious components may be introduced into the shower detection rate (Farley \& Storey 1954). Various methods are used in order to study the dependence of event rate on atmospheric ground pressure, $P$, and temperature, $T$ (Antoni et al. 2004). In a previous paper (Bahmanabadi et al. 2003), we took only the influence of the pressure into account. In the present analysis, the influence of the atmospheric ground pressure, $P$, and temperature, $T$, on the rate of extensive air showers at ground level is taken into account by a second order polynomial (Antoni et al. 2004),

$$
\begin{aligned}
R(P, T)= & R_{0}+\alpha_{1}\left(P-P_{0}\right)+\alpha_{2}\left(P-P_{0}\right)^{2} \\
& +\alpha_{3}\left(T-T_{0}\right)+\alpha_{4}\left(T-T_{0}\right)^{2} \\
& +\alpha_{5}\left(P-P_{0}\right)\left(T-T_{0}\right),
\end{aligned}
$$

The values of $R_{0}=0.107 \mathrm{~s}^{-1}, P_{0}=880 \mathrm{mbar}$, and $T_{0}=18.5^{\circ} \mathrm{C}$ are the long-term mean values of rate, pressure, and temperature, respectively, obtained from the data. The parameters $\alpha_{1}, \alpha_{2}, \alpha_{3}, \alpha_{4}$, and $\alpha_{5}$ were 


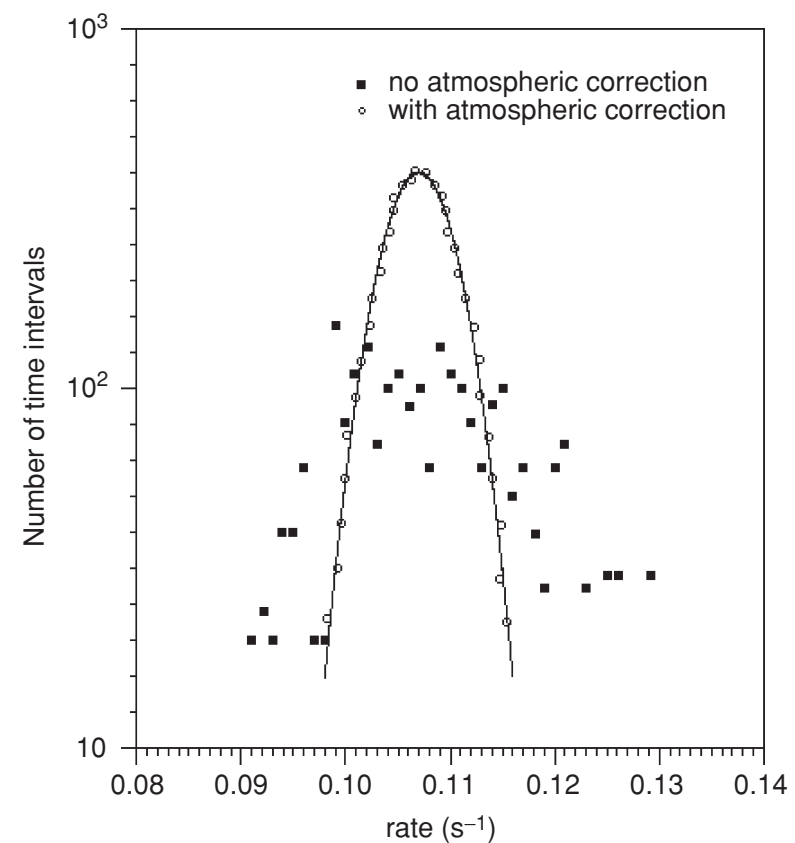

Figure 2 Distribution of rates before and after the atmospheric correction for pressure and temperature. The line curve shows a fit by a Gaussian function.

determined by a fit to the time-dependent rates for the whole observed time, the results being:

$$
\begin{aligned}
& \alpha_{1}=-9.90 \times 10^{-4} \mathrm{~s}^{-1} \mathrm{mbar}^{-1}, \\
& \alpha_{2}=-4.12 \times 10^{-6} \mathrm{~s}^{-1} \mathrm{mbar}^{-2}, \\
& \alpha_{3}=-4.99 \times 10^{-4} \mathrm{~s}^{-1}{ }^{\circ} \mathrm{C}^{-1}, \\
& \alpha_{4}=-4.51 \times 10^{-6} \mathrm{~s}^{-1}{ }^{\circ} \mathrm{C}^{-2}, \text { and } \\
& \alpha_{5}=-5.11 \times 10^{-6} \mathrm{~s}^{-1} \mathrm{mbar}^{-1}{ }^{\circ} \mathrm{C}^{-1} .
\end{aligned}
$$

The correction is done for time intervals of half an hour (in sidereal time $\sim 1795$ SI seconds) by subtracting or adding the necessary number of events calculated by Eqn (3). The quality of the correction can be estimated from Figure 2 which shows the event rate distributions with and without correction for atmospheric ground pressure and temperature. The distribution of uncorrected rates is asymmetric. The distribution of corrected rates is compatible with a Gaussian distribution, as expected for the remaining statistical fluctuation of the event rate.

After the atmospheric correction is made, sidereal time $(S T)$ is calculated using the formula $S T=S T_{0}+\alpha(Z T-$ $Z T_{0}$ ), where $S T_{0}$ can be found in an almanac ${ }^{1}$ for the time $Z T_{0}, Z T$ is the solar time, and $\alpha=1.00273790935$.

Figure 3 shows percentage variation in intensity of the cosmic rays with sidereal time. The data have been fit to Eqn. (4) which describes a curve with first and second harmonics (i.e. with a once-per-day and a twice-per-day variation),

$$
y=A \cos \left[\frac{2 \pi}{24}(t-B)\right]+C \cos \left[\frac{2 \pi}{12}(t-D)\right],
$$

where $t$ is in hours. The results of fitting the data are summarized in Table 1. This analysis shows that the

\footnotetext{
${ }^{1}$ e.g. tycho.usno.navy.mil/sidereal.html
}

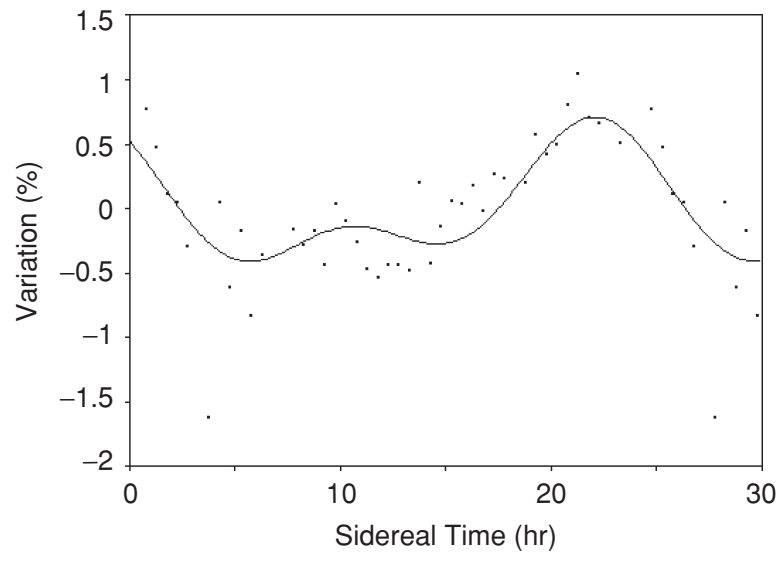

Figure 3 Observed sidereal time variation in intensity of the cosmic rays (points). The curve is the best fit to Eqn. (4) with the coefficients as listed in Table 1.

Table 1. Parameters of the best fit coefficients found for Eqn. (4)

\begin{tabular}{lc}
\hline Amplitude (\%) & Phase (h) \\
\hline$A=0.43 \pm 0.09$ & $B=21.63 \pm 0.77$ \\
$C=0.27 \pm 0.09$ & $D=22.27 \pm 0.63$ \\
\hline
\end{tabular}

anisotropy has a peak close to the sidereal time $21 \mathrm{~h}$, when the zenith is toward the direction of the earth's motion. The amplitude of the anisotropy is of the order of $0.43 \%$. A definite sidereal time variation is seen, whose phase and amplitude are close to those predicted. In order to calculate the magnitude of the anisotropy due to $\mathrm{CGE}$, i.e. the value $\eta$ in Eqn (1), a mean value for $\cos \theta$ is needed (Figure 4). Figure 4 shows the celestial sphere of an observer at latitude $\lambda$. If $\delta$ is the declination of the direction of earth's motion, $\lambda$ the latitude of the observer, and $H$ the hour angle between the observer's meridian and the direction of motion, then the angle $\phi$ between the observer's zenith and the direction of earth's motion is given by

$$
\cos \phi=\sin \delta \sin \lambda+\cos \delta \cos \lambda \cos H,
$$

On the other hand, $\cos \theta$ is calculated by

$$
\cos \theta=\cos \phi \cos \phi^{\prime}+\sin \phi \sin \phi^{\prime} \cos \alpha,
$$

where $\phi^{\prime}$ is the zenith angle of the cosmic ray and $\alpha$ the difference between the azimuth angle of the direction of motion and of the cosmic ray that is, $\alpha=A_{1}-A_{2}$, where $A_{1}$ and $A_{2}$ are obtained from the equations

$$
\begin{gathered}
\sin \delta=\sin \lambda \cos \phi+\cos \lambda \sin \phi \cos A_{1}, \\
\sin \delta^{\prime}=\sin \lambda \cos \phi^{\prime}+\cos \lambda \sin \phi^{\prime} \cos A_{2},
\end{gathered}
$$

where $\delta^{\prime}$ is the declination of the cosmic ray. Following Eqns (5)-(8), the 24-hour mean of the component of cosmic rays in the direction of motion $(\cos \theta)$ may be obtained. The differential zenith angle distribution of cosmic rays can be represented by $\mathrm{d} N=$ constant 


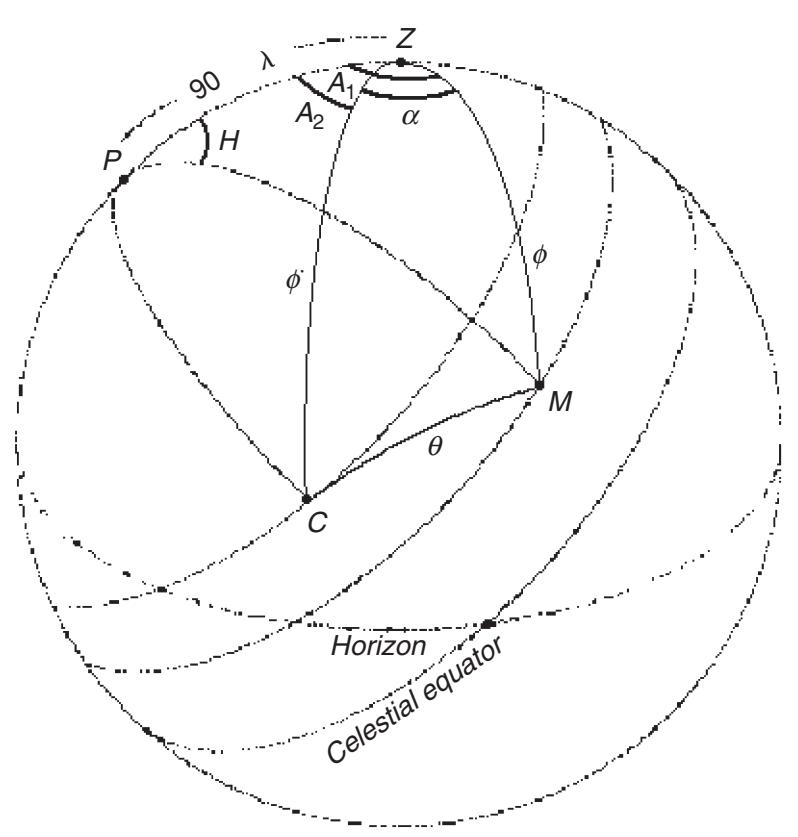

Figure 4 Celestial coordinate, $\mathrm{C}=$ Cosmic ray, $\mathrm{M}=$ direction of earth's motion, $\mathrm{Z}=$ zenith, $\mathrm{P}=$ direction of pole.

$\left(\cos \phi^{\prime}\right)^{n} \sin \phi^{\prime} \mathrm{d} \phi^{\prime}$ with $n=6$; (Khakian et al. 2005). By this distribution, we calculated the mean $\cos \phi^{\prime}$ value which is 0.93 . Also the mean values of $A_{1}$ and $A_{2}$ were obtained by using $\lambda=35^{\circ} 43^{\prime}, \delta^{\prime}=47^{\circ}$, and the mean value of $\delta^{\prime} \approx 30^{\circ}$ (Bahmanabadi et al. 2003). A value of 0.39 is obtained for $\cos \theta$ and this is multiplied by the expected CGE amplitude of $0.345 \%$ to yield a predicted effect of expected value of $0.135 \%$ and the value obtained from experimental data, $0.43 \%$, is about $0.30 \%$. This remaining asymmetry of $0.30 \%$ should have a different origin than CGE.

Since the data were recorded in Tehran at latitude $35^{\circ} 43^{\prime} \mathrm{N}$, the majority of cosmic rays are from the Galactic spiral arm inwards direction, which is at about $20 \mathrm{~h}$ in right ascension and $35^{\circ}$ in declination (Jacklyn 1986). Therefore the remaining asymmetry is probably due to a unidirectional anisotropy of cosmic ray flow along the Galactic arms. A simple diffusion model (Allan 1972) suggests that the value of this asymmetry, $0.30 \%$, would be roughly equal to the ratio of the scattering mean free path to a characteristic dimension of the containment region (i.e. the central Galactic region, with a scale of $10 \mathrm{kpc}$ ). With the amplitude of the anisotropy found in this work being $0.30 \%$, we obtain a mean free path of about $30 \mathrm{pc}$ which is perhaps about 30 gyro radii.

Since the anisotropies are low, it is necessary to consider the effect on the measured counting statistics for a finite data set. If there are $N$ events, then the probability of getting a fractional amplitude greater than $r$ is given by (Linsley 1975),

$$
P(>r)=e^{-k_{0}}, \text { where } k_{0}=r^{2} N / 4,
$$

with $k_{0}$ being a convenient parameter for characterizing the anisotropy amplitude probability distribution. We can take $\sqrt{2} r_{\text {rms }}$, which corresponds to $k_{0}=1$, as the noise amplitude.

For the number of events that we have accumulated, $1.1 \times 10^{6}$, the total amplitude of $0.43 \%$ calculated in this work could have arisen by chance with a probability of $\sim 6.2 \times 10^{-3}$, corresponding to $k_{0}=5.08$ and indicative of an apparently significant anisotropy $\left(k_{0}>1\right)$ at the sidereal period. We conclude therefore that this data set provides evidence of anisotropy of the cosmic ray intensity additional to the CGE.

\section{Conclusion}

Cosmic ray data obtained at the Alborz observatory clearly shows an anisotropy in sidereal time at energies in the decade below $1 \mathrm{PeV}$, with the energy threshold of $\sim 250$ $\mathrm{TeV}$ and the mean energy of $\sim 420 \mathrm{TeV}$. One part of this anisotropy is due to Earth's motion around the Galaxy (the CGE), but our measured asymmetry suggests the possible existence of some other additional effects, probably a unidirectional anisotropy of cosmic ray flow along the Galactic arms. The amplitude of our total measured anisotropy is about $0.43 \%$. The CGE contribution to this anisotropy is about $0.135 \%$ and the rest, $0.30 \%$, is predicted to be due to the flow along the Galactic arm. The latter anisotropy suggests a mean free path of about $30 \mathrm{pc}$ for these high-energy cosmic rays. The evidence for these anisotropies is based on the value of the parameter $k_{0}$, as suggested by Linsley (1975) and found in this work to be 5.08, that is, more than $k_{0}=1$, the value for the noise amplitude.

The EAS-TOP experiment reported somewhat lower limits in the energy range below $1200 \mathrm{TeV}$ (Aglietta et al. 2003). The relatively large amplitudes published by the Akeno experiment (Kifune et al. 1986) and our experiment are difficult to reconcile with the results of the EAS-TOP experiment.

\section{Acknowledgements}

This research has been partly supported by Grant No. NRCI 1853 of the National Research Council of the Islamic Republic of Iran.

\section{References}

Aglietta, M., et al. 1996(EAS-TOP Collaboration) 1996, ApJ, 470, 501 Aglietta, M., et al. (EAS-TOP Collaboration) 2003, Proc. 28th ICRC, Tsukuba, Japan, HE 1.1, 183, edited by T. Kajita, Y. Asaoka, A. Kawachi, Y. Matsubara, M. Sasaki, Tokyo, University Academy Press, Inc.

Allan, H. R. 1972, ApJL, 12, 237

Antoni, T. et al. 2004, ApJ, 604, 687

Bahmanabadi, M. et al. 1998, ExA, 8, 211

Bahmanabadi, M. et al. 2003, ExA, 15, 13

Clay, R. W., McDonough, M. A. \& Smith, A. G. K. 1997, Proc. 25th ICRC, Durban, South Africa, 4, 185

Compton, A. H. \& Getting, I. A. 1935, PhRv, 47, 817

Farley, F. J. M. \& Storey, J. R. 1954, PPS, A67, 996

Gaisser, T. K. 1990, in Cosmic Rays and Particle Physics (New York: Cambridge University Press)

Heck, D. et al. 1998, Report FZKA 6019, Forschungszentrum Karlsruhe

Jacklyn, R. M. 1986, PASA, 6, 425 
Khakian Ghomi, M., Bahmanabadi, M. \& Samimi, J. 2005, A\&A, 434, 459

Kifune, T. et al. 1986, J. PhG, 12, 129

Lin, T. F., Fields, B., Gress, J. \& Poirier, J. 1999, Proc. 26th ICRC, Salt Lake city, Utah, USA, HE3.2.09, 100, edited by D. Kieda, M. Salamon, and B. Dingus, High Energy Astro Physics institute University of Utah
Linsley, J. 1975, PhRvL, 34, 1530

Poirier, J., D'Andrea, C. \& Dunford, M. 2001, Proc. 27th ICRC, Hamburg, Germany, 3930, edited by K. H. Kampert, G. Hainzelmann, and C. Spiering, Copernicus systems and Technology GmbH, Berlin, Germany

Smith, A. G. K. \& Clay, R. W. 1997, AuJPh, 50, 827 